\title{
Role of human milk in the development of gastrointestinal bacterial flora and immunity in preterm infants
}

\begin{abstract}
The intestinal microflora plays an important role in the health and well-being of the host. Crucial to this is the provision of a natural defense mechanism against invading pathogens. Intestinal flora contains both friendly and potentially pathogenic organisms. Well-balanced bacterial colonization is an important factor in the development and maintenance of the gut immune system. Among the factors influencing the establishment of the intestinal flora, age, diet, medications, and stress appear to be the leading causes.

The intestinal flora of the newborn plays a major role in the development of gut physiology and pathology leading to gut immunology and the ability to fight neonatal infections and other gastrointestinal diseases.

Full term infants receive regular human or non-human milk diets orally as their primary nutritional source. This facilitates the normal intestinal colonization process. Preterm infants lack this feeding and therefore are deprived of the host defense mechanisms influenced by gut flora. This review discusses the factors in human milk that contribute to gut physiology, gut microbiology, and intestinal immunity, which lead to the healthy growth of preterm infants.
\end{abstract}

Keywords: human milk, preterm infants, nutrition, gastrointestinal flora, immunity
Volume I Issue 4 - 2014

\section{Champa N Codipilly Codipilly}

Division of Neonatal/Perinatal Medicine, Cohen Children's

Medical Center, USA

\begin{abstract}
Correspondence: Champa N Codipilly Codipilly, The Lilling Family Neonatal Research Laboratory, Division of Neonatal/ Perinatal Medicine, Cohen Children's Medical Center at North Shore-LIJ Health System, Manhasset, NY and Feinstein Institute for Medical Research, NS-LIJ Health System, Manhasset, NY I 1030, USA, Tel 516-562-3567, Email ccodipil@nshs.edu
\end{abstract}

Received: July 30, 20I4 | Published: September 13, 2014
Abbreviations: GIT, gastrointestinal tract; GALT, gutassociated-lymphoid-tissue; VLBW, very low birth weight; TPN, total parenteral nutrition; NEC, necrotizing enterocolitis; NICU, neonatal intensive care unit; sIgA: secretory $\operatorname{IgA}$

\section{Intestinal Immune System}

One of the key immune organs of the body is the gastrointestinal tract (GIT). The intestinal immune system is associated with several types of lymphoid organs and is collectively referred to as the gutassociated-lymphoid-tissue (GALT). ${ }^{1}$ This consists of Peyer's patches, mesenteric lymph nodes, isolated lymphoid follicles, cryptopatches, lymphocytes, and dendritic cells in the lamina propria and intestinal epithelium. Peyer's patches and mesenteric lymph nodes are considered secondary lymphoid tissues and these develop during fetal life. Other structures, known as tertiary lymphoid tissue, develop post-natally. ${ }^{2}$ The intestinal epithelial cells maintain tight junctions, producing a physical barrier against the penetration of pathogens. ${ }^{3}$ GALT is considered the largest collection of lymphoid tissues in the body. ${ }^{4}$

With the presence of close to $80 \%$ of the antibody producing cells, the GIT is the major antibody producer of the body. ${ }^{5}$ The intestinal immune system is designed to act as a buffer by monitoring exposure of pathogenic bacteria to the host. For this reason, the mucosal immune system is continuously stimulated by GI microbes. ${ }^{6}$ Hakansson and Molin ${ }^{4}$ suggests that microbial colonization of the GI tract affects the composition of GALT. Once the intestine is exposed to microorganisms, intraepithelial lymphocytes increase; germinal centers with immunoglobulin-producing cells arise rapidly in follicles and in the lamina propria and serum immunoglobulin concentrations increase substantially. Host-microbe interactions in the gut are wellbalanced; secretory $\operatorname{IgA}$, the predominant immunoglobulin found in the intestinal epithelium, is produced by plasma cells in the lamina propria and transported to the lumen by a poly immunoglobulin receptor. Goblet cells in the intestinal epithelium secrete a protective mucus layer, which limits the contact between bacteria and epithelial cells. Paneth cells residing in the crypts of the small intestine secrete bactericidal peptides. Epithelial cells and the mucosal immune system are capable of distinguishing pathogenic and non-pathogenic organisms in the gut. These cells detect bacterial antigens and initiate and regulate both innate and adaptive immune responses. ${ }^{1,7,8}$

\section{Initial acquisition of infant gut bacteria}

The infant gut is sterile in utero and initially bathed only in swallowed amniotic fluid. The GIT of a newborn baby is colonized during the birth process and thereafter from the mother and surrounding environment until a dense population of microorganisms is established. The mode of child delivery (vaginal or caesarian), infant diet, infant care site (hospital nursery, parental home) and maternal or infant use of antibiotics are some factors that play an important role in establishing the intestinal flora. A caesarean birth, which involves a surgical procedure, could introduce different microorganisms than those found in the vaginal canal. ${ }^{1,9-11}$ Usually, the bulk of the intestinal microbiota originates in the mouth, which harbors various species, and which is fed to the intestinal channel by the swallowing reflex. Establishing the gut flora during the first year of life is a complex ecological process that includes the intestinal mucosa of the GI tract and more than 400 interdependent species of bacteria. ${ }^{9}$

The stability of the gut ecosystem depends on the dynamic balance between the GI tract bacterial flora, host physiology, and $\operatorname{diet}^{12}$. Many studies have shown that breast-fed babies have different fecal microbial profiles than those are bottle-fed. The intestinal flora of human milk fed babies is predominantly colonized with Bifidobacteria and Lactobacilli in addition to Staphylococci, Streptococci, Corynebacteria, Propionibacteria, and Micrococci. 
These enter through contact of the nipple, milk ducts, and surrounding skin during feeding. The most prevalent organisms in the gut of formula fed infants are Staphylococci, Streptococci, Bacteroides, and Clostridium species. ${ }^{13}$ In general, intestinal bacterial flora falls into three possible categories-beneficial, potentially pathogenic, and pathogenic. In the intestine, non-pathogenic bacteria inhibit the growth of pathogenic bacteria, providing a balanced, but complex, ecosystem. ${ }^{14}$

\section{Bacterial flora of a preterm infant}

Even though information on the development of intestinal microbial flora of term infants is available, such data are scarce for preterm infants. Studies have used both culture techniques and molecular methods to investigate preterm infant intestinal flora. According to Dai and Walker, ${ }^{15}$ Enterobacteria and Streptococci are the first microorganisms to colonize the intestine of very low birth weight (VLBW) infants and these will predominate in the long term. Bifidobacteria appear in the stools of VLBW infants at a mean age of 10 days and become the predominant organism at 20 days. In contrast, in breast-fed full term infants Bifidobacteria appear at about 4 days. VLBW infants acquire bowel organisms at a slower rate, particularly during the time they are fed parenterally. While in the NICU, VLBW infants had only one or few species of organisms present in their feces. However, these organisms appear to exist in much greater numbers than in normal infants, who have a more balanced intestinal makeup. In adults, the species composition of luminal flora does not change from the ileocecal valve to the anus. ${ }^{16}$ Comparatively, during the first weeks of oral feeding in the NICU, VLBW infants showed a spontaneous overgrowth of organisms in the distal portion of the bowel. Frequent isolation of $S$. epidermidis in the feces of preterm infants in the NICU has been reported when fed human milk. ${ }^{15}$

It is reported that low birth weight neonates who are at high risk of developing gastrointestinal disorders receive better protection when fed human milk than formula. ${ }^{17}$ However, human milk itself may not be a source of complete nutrition for infants born at $<32$ weeks $(<1500 \mathrm{~g})$. Commonly, milk fortifiers are added to their mother's milk. If the infant is born at $<28$ weeks, total parenteral nutrition (TPN) is necessary during the first two to three weeks until the baby reaches full enteral intake. Since TPN does not contain most milk ingredients, its influence on establishing gut flora is more adverse when compared to full term infants who are fed human milk. ${ }^{18,19}$

Often, preterm babies need medical attention requiring antibiotics; these antibiotics can alter their intestinal micro flora. Antibiotics taken by the mother can pass to the baby through breast milk and affect the infant's intestinal flora. It has been shown that antibiotic therapy disturbs the intestinal flora with a resulting decrease of normal anaerobic bacteria and an increase in the growth of potential pathogens such as yeast, Pseudomonas, and Clostridia, as well as the possible inducement of antibacterial resistance..$^{20}$

Bacterial colonizing patterns becomes progressively more normal as the diversity of colonizing species increases in later weeks, which can be altered by the administration of broad-spectrum antibiotics. ${ }^{13}$ In recent years, probiotic and prebiotic substances have been used to change or alter the microbial flora. ${ }^{21,22}$

\section{Human Milk Factors Influencing Bacterial Growth and Immunity in Intestines}

Even though many studies have investigated the differences in fecal flora between breast-fed and formula-fed infants, the mechanisms that lead to these differences are not well understood. Dai and Walker ${ }^{15}$ have summarized these changes as relating to the type of proteins, the availability of iron, the presence of oligosaccharides, and the $\mathrm{pH}$ of milk. The fecal flora of infants who were fed wheybased formulas had similarities to the floras of breast-fed infants. Both infant formula and human milk contain iron. Human milk contains lactoferrin, an iron binding glycoprotein. The availability of iron plays a significant role, whether formula or human milk. The predominant organisms, Bifidobacteria and Lactobacilli, found in breast fed infants do not need iron for their growth, whereas bacteroides species and other Enterobacteria do require iron. It is also suggested that lactoferrin supplementation may suppress the growth of facultative organisms in the infant gut.

Lactoferrin exerts its antibacterial activity in two different ways. It binds to iron and deprives microorganisms of iron needed for growth while directly damaging the outer membrane of gram negative bacteria. ${ }^{23}$ Lactoferrin is also a major protein in human milk and once digested it contributes to the amino acid pool of the human gut. These amino acids are an important nutritional component absorbed by breast-fed infants. Fat is the major source of calories and constitutes approximately $4 \%$ of human milk. Soon after milk consumption, fatty acids and monoglycerides, the digestion products of fat, can be found in high concentrations in the intestine. ${ }^{24}$

Oligosaccharides are the third largest solid component comprising about $1 \%$ of human milk. They are largely indigestible and pass through the infant's intestinal tract. However, because they provide more than $5 \%$ of the calories of human milk, it is assumed that these may provide an essential non-nutritive function to the infant. ${ }^{24}$ Some $\mathrm{N}$-acetyl glucosamine containing oligosaccharides in breast milk are shown to be "growth factors" for Bifidobacteria and Lactobacilli.

The buffering capacity of human milk also plays an important role in acid-base balance. Its buffering capacity is lower compared to cow's milk and formula milk. Bifidobacteria and Lactobacilli, which require a lower $\mathrm{pH}$ for growth, appear to thrive when human milk is present. At the same time, a lower $\mathrm{pH}$ seems to inhibit other pathogenic bacteria. It is possible that this feature of human milk helps maintain an acidic gut $\mathrm{pH}$ and enhances the growth of beneficial bacteria. ${ }^{15}$

Human milk contains both micro and macronutrients. The micronutrients in human milk include vitamins A, B1, B2, B6, B12, D, iodine and fatty acids. ${ }^{25}$ These micronutrients play an important role in systemic immunity, and deficiencies could lead to immunosuppression and/or immune dysregulation. ${ }^{26}$

The macronutrients include protein, fat, and lactose ${ }^{25}$ Specific proteins include bile salt-stimulated lipase (aids in digestion of lipids in newborns, especially in preterm infants who have low lipase activity and poor lipid utilization), amylase (active at low $\mathrm{pH}$, may compensate for low salivary and pancreatic amylase activity in newborns and helps in the digestion of complex carbohydrates), $\beta$-casein (a highly phosphorylated protein, which forms phosphopeptides that keeps $\mathrm{Ca}++$ soluble and aids in its absorption), lactoferrin (facilitates the absorption of iron by intestinal cells in vitro), haptocorrin (vitamin B-12 is bound to haptocorrin and facilitates the absorption of the vitamin early in life), and $\alpha 1$-antitrypsin (a protease inhibitor which may limit the activity of pancreatic enzymes in breast-fed infants). All of these proteins help in the digestion and utilization of micro and macronutrients from the milk. ${ }^{27}$

According to Fanaro et al., ${ }^{28}$ the viability of intestinal bacteria is 
affected by the availability of substrates/nutrients, redox potential, $\mathrm{O} 2$ tension, $\mathrm{pH}$, and bacterial colonization sites in the colon.

Human milk also provides immunologic advantages for low birth weight as well as term born infants. ${ }^{29}$ Studies have shown that preterm infants fed human milk have advantages over their formula fed counterparts. Antimicrobial and immunologic components of human milk give infants the protection against infection and the opportunity to have a healthy growth pattern. This renders them less prone to conditions such as life threatening necrotizing enterocolitis (NEC) urinary tract infections, otitis media, and late-onset sepsis..$^{30}$ A study by Kramer et al., ${ }^{31}$ had shown that a lower risk of gastrointestinal infections was associated with exclusively breastfed infants for the first 6 months of life, and these infants had no demonstrable adverse health effects in their first year of life.

Newborn infants lack fully developed immune systems. Though structures of the immune system are developed in utero by 28 weeks of gestation and completed at the time of birth, their activation does not occur until after birth. ${ }^{32}$ Most full term newborn babies receive breast milk as their sole diet during their first 4 to 6 months of life. It is assumed that human milk is nutritionally superior and has the ability to aid in digestion, help maturation of the gastrointestinal tract, and express numerous host defense properties. ${ }^{33}$ These include maternal antibodies, maternal cytokines, leukocytes, chemokines, and hormones. ${ }^{34}$

Human milk also contains components that inhibit pathogenic bacterial growth while providing a prebiotic effect that stimulates the colonization of essential non-pathogenic gut bacteria. These include, but are not limited to, secretory antibodies, multifunctional agents such as fatty acids and lactoferrin, homologues of host cell surface glycoconjugates (carbohydrate moieties such as glycoproteins, glycolipids, glycosaminoglycans, and mucins, ${ }^{24,35}$ hormones, peptides, and amino acids.

Liepke et $\mathrm{al}^{36}$ identified peptides in human milk that selectively stimulate the growth of Bifidobacteria, including a milk component called bifidus factor which stimulates the growth of Bifidobacterium bifidum. Other bifidogenic factors include oligosaccharides, which are small chain carbohydrates consisting of lactose at the reducing end and $\mathrm{N}$-acetylglucosamine, glucose, fucose, or sialic acid at the nonreducing terminus. Of note, colostrum, the earliest milk expressed from nursing mothers, has higher oligosaccharide content than mature milk. ${ }^{35,37}$

Nucleotides are another component present in human milk that is minimally present in cow's milk, of which the majority of infant formulas are derived. It has been observed the nucleotides are cofactors the augment the growth of Bifidobacteria in vitro. However, the addition of nucleotides to infant formula did not result in an enhancement of Bifidobacteria in fecal flora. ${ }^{38}$

Lysozyme, an enzyme found in the human milk whey fraction, is capable of degrading the outer cell wall of gram positive bacteria. It is also capable of killing gram negative bacteria in vitro synergistically with lactoferrin. ${ }^{28}$

Oligosaccharides present in milk provide an essential non-nutritive function to infants. ${ }^{24}$ Studies have shown that oligosaccharides in human milk inhibit adherence of pathogens to target cells. Accordingly it inhibits binding of Streptococcus pneumoniae, enteropathogenic Escherichia coli, invasive strains of Clostridium jejuni, and the toxicity of heat stable toxin of E. coli. ${ }^{35}$
Secretory $\operatorname{Ig} \mathrm{A}(\mathrm{s} \operatorname{Ig} \mathrm{A})$ is the major type of immunoglobulin (>90\%) found in human milk. It is relatively resistant to intestinal proteolysis. In the early lactation period, $\operatorname{sgA}$ is found in high concentrations in milk (1-2 g/L), but levels taper down $(0.5-1 \mathrm{~g} / \mathrm{L})$ after2 years of lactation. In breast-fed infants modest amounts of sIgA can be found intact in their stools. By breast-feeding, mothers can transfer their immunity against enteric pathogens in the form of sIgA, allowing the infant's immature immune system to be boosted by this acquired antibody. ${ }^{25}$ The presence of SIgA has been considered the cause for the lower incidence of enteric disease among breast-fed infants. Leukocytes and macrophages are also found in large numbers in human milk and provide acquired immunity to the infant. ${ }^{22}$

According to Fituch et al. ${ }^{39}$ the anti-inflammatory cytokine IL-10 is present in both term and preterm human milk in similar concentrations. The study also found that IL-10 was not detectable at significant levels in the breast milk of mothers of infants who developed NEC, suggesting a protective role for this cytokine. The exact function of IL-10 in human milk on immunity is not well understood. However, studies have shown that IL-10 gene-deficient neonatal mice were afflicted with enterocolitis at a greater rate in comparison to the control group.

\section{Pre/Probiotic Use in Altering GI Flora}

NEC is considered the most common gastrointestinal emergency in the neonatal intensive care unit (NICU) and is a major cause of morbidity in preterm infants. The exact etiology and pathogenesis have not been completely understood. The most common risk factors cited are prematurity, asphyxia, intestinal ischemia, and enteral feeding. ${ }^{39}$ Other risk factors include immaturity of mucosal host defense mechanisms, inappropriate bacterial colonization, an imbalance of endothelin-dependent vasoconstriction, and nitric oxide dependent vasodilation. ${ }^{39-41}$ According to published literature, bacterial colonization in the intestine greatly depends on the type and substance of infant feed. ${ }^{35,38,42}$

Bifidobacteria are the predominant organism colonized in the breast-fed infant's intestine and contributes to the protection against intestinal infections. Bifidobacteria have numerous potential beneficial properties including inhibiting the growth of potential pathogens, lowering blood ammonia levels, producing vitamin B and folic acid, promoting immunologic attack against malignant cells, improving host resistance to pathogens, producing nutrients for colonocytes, reducing blood cholesterol levels, and restoring the normal microflora during antibiotic therapy. ${ }^{15}$ The formula-fed infant's intestinal flora is a complex mixture of bifidobacteria, bacteroides, clostridia, and anaerobic streptococci. ${ }^{16,28,40}$ Therefore, one can postulate that these infants do not have the total benefits that breast-fed infants have with a predominantly bifidobacteria flora. Attempts have been made to alter this flora to one that is more bifidobacteria predominant using probiotics and prebiotics. ${ }^{19,22,41}$

\section{Conclusion}

Nutrition is key for the healthy growth and development of newborns, and it is clear that breast milk seems to have no match for nutritional sustenance for the newborn. However, there is more to breast milk than simple nutrition. The role of breast milk in establishing gut flora and initiating immune protective mechanisms are equally important to the newborn's development and health. Evidently, these are two aspects affecting the health of preterm infants, who are often introduced to breast milk later in life. 
GI microbial ecology in health is at a dynamic equilibrium, consisting of various microbial groups that require vivid growth conditions. The local environmental conditions of the gut such as $\mathrm{pH}$ and hydration status are determined mainly by the bacterial metabolic activity. The major source of substrate for the gut microbes is host-ingested food. Therefore, the diet plays an important role in determining the GI flora. The wide array of proteins, carbohydrates, lipids, and minerals in breast milk provides infants with essential nutrition. Lactose is one sugar that many lactobacilli metabolize leading to the formation of acid end products such as lactic and acetic acids, which lowers the local $\mathrm{pH}$. This low $\mathrm{pH}$ creates favorable conditions for bifidus and other lactobacilli species to thrive while suppressing non-acidophilic organisms, establishing a unique microbial environment.

A lack or delay in breast milk feeding has an impact on nutrition, immunity, and the establishment of a healthy intestinal flora. Deprivation of colostrum, which is rich in immune and nutritional components, at this early stage of life, is a challenge for the healthy growth of preterm infants. To the contrary, exposure to maternal antibiotics through breast milk could also have adverse effects on GI flora. Interpreting the current evidence available, it could be hypothesized that appropriate dietary changes and enhancement of GI probiotic evolution may boost preterm nutrition, health, and survival. New studies using novel technologies for evaluating the human microbiome will help determine the factors that contribute to the development of the gut flora and healthy immune system in infants ${ }^{42}$

\section{Acknowledgements}

None.

\section{Conflicts of Interest}

There is no conflict of interest.

\section{Funding}

None.

\section{References}

1. Sommer F, Backhed F. The gut microbiota-masters of host development and physiology. Nat Rev Microbiol. 2013;11(4):227-238.

2. Littman DR, Pamer EG. Role of the commensal microbiota in normal and pathogenic host immune responses. Cell Host Microbe. 2011;10(4):311-323.

3. Magalhaes JG, Tattoli I, Girardin SE. The intestinal epithelial barrier: how to distinguish between the microbial flora and pathogens. Semin Immunol. 2007;19(2):106-115.

4. Hakansson A, Molin G. Gut Microbiota and Inflammation. Nutrients. 2011;3(6):637-682.

5. Ouwehand A, Isolauri E, Salminen S. The role of the intestinal microflora for the development of the immune system in early childhood. Eur $J$ Nutr. 2002;41(Suppl 1):32-37.

6. Brown EM, Sadarangani M, Finlay BB. The role of the immune system in governing host-microbe interactions in the intestine. Nat Immunol. 2013;14(7): 660-667.

7. Chung H, Kasper DL. Microbiota-stimulated immune mechanisms to maintain gut homeostasis. Curr Opin Immunol. 2010;22(4): 455-460.

8. Spor A, Koren O, Ley R. Unravelling the effects of the environment and host genotype on the gut microbiome. Nat Rev Microbiol. 2011;9(4):279-290.
9. Kelly D, King T, Aminov R. Importance of microbial colonization of the gut in early life to the development of immunity. Mut Res. 2007;622(12):58-69.

10. Clemente JC, Ursell LK, Parfrey LW, et al. The impact of the Gut Microbiota on Human Health: an Integrative View. Cell. 2012;148(6): $1258-1270$.

11. Dominguez-Bello MG, Blaser MJ, Ley RE, Knight R. Development of the human gastrointestinal microbiota and insights from highthroughput sequencing. Gastroenterology. 2011;140(6):1713-1719.

12. Lozupone CA, Stombaugh JI, Gordon JI, et al. Diversity, stability and resilience of the human gut microbiota. Nature. 2012;489(7415): 220 230

13. Thompson-Chagoyan OC, Maldonado J, Gil A. Colonization and impact of disease and other factors on intestinal Microbiota. Dig Dis Sci. 2007;52(9):2069-2077.

14. Westerbeek EA, van den Berg A, Lafeber HN, et al. The intestinal bacterial colonization in preterm infants: A review of the literature. Clin Nutr. 2006;25(3):361-368.

15. Dai D, Walker WA. Protective nutrients and bacterial colonization in the immature human gut. Adv Pediatr. 1999;46: 353-382.

16. Bennet R, Eriksson M, Nord CE, et al. Fecal bacterial microflora of newborn infants during intensive care management and treatment with five antibiotic regimens. Pediatr Infect Dis. 1986;5(5):533-539.

17. Dvorak B, Fituch CC, Williams CS, et al. Increased epidermal growth factor levels in human milk of mothers with extremely premature infants. Pediatr Res. 2003;54(1):15-19.

18. Schanler RJ. The use of human milk for premature infants. Pediatr Clin North Am. 2001;48(1):207-219.

19. Bakewell-Sachs S, Blackburn S. State of the Science: Achievements and Challenges across the Spectrum of Care for Preterm Infants. $J$ Obstet Gynecol Neonatal Nurs. 2003;32(5):683-695.

20. Mathew JL. Effect of maternal antibiotics on breastfeeding infants. Postgrad Med J. 2004;80(942):196-200.

21. Nair V, Soraisham AS. Probiotics and prebiotics: role in prevention of Nosocomial Sepsis in Preterm Infants. Int J Pediatrics. 2013.

22. Patel RM, Denning PW. Therapeutic use of prebiotics, probiotics and postbiotics to prevent necrotizing enterocolitis: what is the current evidence? Clin Perinatol. 2013;40(1):11-25.

23. Yamauchi K, Tamita M, Giehl TJ, et al. Antibacterial activity of lactoferrin and a pepsin-derived lactoferrin peptide fragment. Infect Immun. 1993;61(2):719-728.

24. Newberg DS. Bioactive components of human milk. Evolution, efficiency and protection. Adv Exp Med Biol. 2001;501:3-10.

25. Ballard O, Morrow AL. Human milk composition: nutrients and bioactive factors. Pediatr Clin North Am. 2013;60(1):49-74.

26. Spencer SP, Belkaid Y. Dietary and commensal derived nutrients: Shaping mucosal and systemic immunity. Curr Opin Immunol. 2012;24(4):379-384.

27. Lonnerdal B. Nutritional and physiologic significance of human milk proteins. Am J Clin Nutr. 2003;77(6):1537S-1543S.

28. Fanaro S, Chierici R, Guerrini $\mathrm{P}$, et al. Intestinal microflora in early infancy: composition and development. Acta Paediatr Suppl. 2003;91(441):48-55.

29. Schanler RJ, Schulman RJ, Lau C. Feeding strategies for premature infants: Beneficial outcomes of feeding fortified human milk versus preterm formula. Pediatrics. 1999;103(6):1150-1157. 
30. Gartner LM, Morton J, Lawrence RA, et al. American Academy of Pediatrics policy statement: Breastfeeding and the use of human milk. Pediatrics. 2005;115(2):496-506.

31. Kramer MS, Guo T, Platt RW, et al. Infant growth and health outcomes associated with 3 compared with 6 months of exclusive breastfeeding. Am J Clin Nutr. 2003;78(2):291-295.

32. Gleeson M, Cripps AW. Development of mucosal immunity in the first year of life and relationship to sudden infant death syndrome. FEMS Immunol Med Microbiol. 2004;42(1):21-33.

33. Schanler RJ, Garza C, Nichols B. Fortified mother's milk for very low birth weight infants: Results of growth and nutrient balance studies. $J$ Pediatr. 1985;107(3):437-445.

34. Palmer AC. Nutritionally mediated programming of the developing Immune system. Adv Nutr. 2011; 2(5):377-395.

35. Newberg DS. Oligosaccharides in human milk and bacterial colonization. J Pediatr Gastroenterol Nutr. 2000;30(suppl 2):S8-S17.

36. Liepke C, Adermann K, Raida M, et al. Human milk provides peptides highly stimulating the growth of bifidobacteria. Eur J Biochem. 2002;269(2):712-718.
37. Edwards CA, Parrett AM. Intestinal flora during the first months of life: New perspectives. Br J Nutr. 2002;88(Suppl 1): S11-S18.

38. Balmer SE, Hanvey LS, Wharton BA. Diet and faecal flora in the newborn: nucleotides. Arch Dis Child Fetal Neonatal Ed. 1994;70(2): F137-F140.

39. Fituch CC, Palkowetz KH, Goldman AS, et al. Concentrations of IL10 in preterm human milk and in milk from mothers of infants with necrotizing enterocolitis. Acta Paediatr. 2004;93(11):1496-1500.

40. Hoyos AB. Reduced incidence of Necrotizing Enterocolitis associated with enteral administration of Lactobacillus acidophilus and Bifidobacteriuminfantis to neonates in an intensive care unit. Int J Infect Dis. 1999;3(4):197-202.

41. Dani C, Biadaioloi R, Bertini G, et al. Probiotics feeding in prevention of urinary tract infection, bacterial sepsis and necrotizing enterocolitis in preterm infants. Biol Neonate. 2002;82(2):103-108.

42. La Rosa PS, Warner BB, Zhou Y, et al. (2014) Patterned progression of bacterial populations in the premature infant gut. Proc Natl Acad Sci USA. 2014;111(34):12522-12527. 